\title{
Rapid extinction of conditioned reinforcement effects in the signaled absence of primary reinforcement
}

\author{
JOHN C. BIRKIMER and CHARLES E. \\ AYLWORTH, University of Louisville, \\ Louisville, Ky. 40208
}

Six male albino rats were trained on a multiple schedule involving intermittent white noise and sucrose solution for bar-pressing in the presence of one stimulus and extinction in the presence of the second stimulus. When responding in the second stimulus context reached a low level, the noise was made available for each response in that context. The noise functioned as a conditioned reinforcer, temporarily raising response rates in the second stimulus context above their earlier level. The reinforcing effects of the noise quickly extinguished, suggesting procedural or species-specific differences between the current study and recent research with pigeons.

Several authors have criticized the traditional resistance-to-extinction tests of conditioned reinforcement (Kelleher \& Gollub, 1962; Kelleher, 1966; Zimmerman \& Hanford, 1966) because they test for conditioned reinforcing effects after primary' reinforcement has been eliminated from the experimental situation. Further, Zimmerman and Hanford suggested that the extinction tests do not model nonexperimental situations in which the concept of conditioned reinforcement is often invoked. Another obvious criticism of those techniques is that they confound the extinction of a response and the extinction of the conditioned reinforcer. Consequently, such studies must compare the extinction responding of two groups of Ss, one that receives the supposed conditioned reinforcer for responding and one that does not.

As an alternative to the extinction tests of conditioned reinforcement, Kelleher \& Gollub (1962) recommended the study of stimulus functions in chained schedules, Kelleher (1966) suggested the study of brief stimuli in second-order schedules, and Zimmerman \& Hanford (1966) presented a third alternative, the study of response-produced stimuli occasionally paired with response-independent food. The present paper examined a fourth technique, the study of conditioned reinforcement in an extinction component of a multiple schedule. 1
Skinner (1938) demonstrated a conditioned-reinforcement test procedure that involved first partially extinguishing responding without the supposed conditioned reinforcing stimulus and then making the stimulus available for responding, thus producing a second extinction curve more clearly reflecting the strength of the conditioned reinforcer independently of the strength of the conditioned response. The procedure does, however, eliminate primary reinforcement from the testing situation.

Multiple schedules of reinforcement (Ferster \& Skinner, 1957) are those involving two or more independent reinforcement schedules, only one of which is in effect at a given time, and each of which has its own correlated stimulus condition. A multiple schedule, in which a neutral stimulus and primary reinforcement remain available in one stimulus condition and the procedure developed by Skinner is used in the other stimulus condition, would seem to satisfy the objections to extinction tests discussed above. Primary reinforcement would be maintained in the experimental situation, the conditioned reinforcer would be tested after the conditioned response is extinguished in the second stimulus condition, and the procedure seems analogous to situations in which the concept of conditioned reinforcement is often used as an explanatory device.

The current study, then, tested for conditioned reinforcing effects in a multiple schedule in which, during the first stimulus condition, responding produced a white noise and primary reinforcement, and, during the second stimulus condition, responding was first extinguished to a low level and then produced only the noise.

\section{METHOD}

The Ss were six male Sprague-Dawley rats, about 120 days old at the beginning of the study. A commercially manufactured operant-conditioning apparatus was used (Lehigh Valley 1316), with events and recording controlled by electromechanical equipment in an adjacent room.

The Ss were placed on 23 th food deprivation and trained during daily 1 -h sessions. They were magazine trained, shaped to bar-press, and given 3 days of continuous reinforcement training, with a 1-sec white noise immediately preceding each $.01 \mathrm{cc}$ of $30 \%$ sucrose solution reinforcement for each bar press. The noise and reinforcement were then presented for responding on a variable-interval schedule (VI 20-sec) for 5 days (Rats 1,2, and 3) or 3 days (Rats 4, 5, and 6). After this training was completed, a multiple schedule was introduced. During the Stimulus 1 (SI) condition, houselight on, a continuation of the previous procedure, the noise and subsequent sucrose delivery were still available for responding on the VI 20-sec schedule. During the Stimulus 2 (S2) condition, houselight off, responding produced neither noise nor sucrose solution (EXT). S1 periods were of fixed 1-min duration and $S 2$ periods of variable duration, with a mean of $1 \mathrm{~min}$. This multiple schedule (Mult VI 20-sec EXT) with the $\mathrm{S} 2$ extinction component was continued until each rat consistently emitted at least 10 times as many responses in $\mathrm{S} 1$ as in S2. This required 12 days for Rat 1, 15 days for Rats 2 and 3, and 18 days for Rats 4, 5, and 6.

When $S 2$ rates were thus consistently low, the $\mathbf{S 2}$ schedule was modified so that responses in $S 2$ now produced the white noise on a continuous reinforcement schedule (CRF:S), $1 \mathrm{sec}$ of noise for each S2 response. No sucrose solution was presented in S2, but the noise and sucrose solution remained available for responding on the VI 20-sec schedule in S1. Conditioned reinforcing effects of the white noise should produce an increase in S2 response rates under this schedule (Mult VI 20-sec CRF:S).

After 8 days of this last schedule, the rats were returned to the original multiple schedule for 2 days to assess possible changes in the baseline rate in S2. Two rats received continued training; Rat 5 was maintained on the Mult VI 20-sec EXT schedule for 6 additional days, and Rat 4 was maintained on that schedule for 2 extra days and then returned to the Mult VI 20-sec CRF:S schedule for a final 4 days.

\section{RESULTS}

Figure 1 shows the response rates for each rat in the $\$ 2$ condition across the last 5 days under the original multiple schedule (Days -5 through -1 ), the 8 days under the Mult VI 20-sec CRF:S schedule with the noise available in S2 (Days 0 through 7 ), the final 2 days of exposure to the original multiple schedule (Days 8 and 9), and the additional training of Rats 4 and 5 . The change in the consequences of S2 responding had an immediate effect; on the first day with noise available for responding in $\mathbf{S 2}$, mean response rates in that condition were more than triple the 

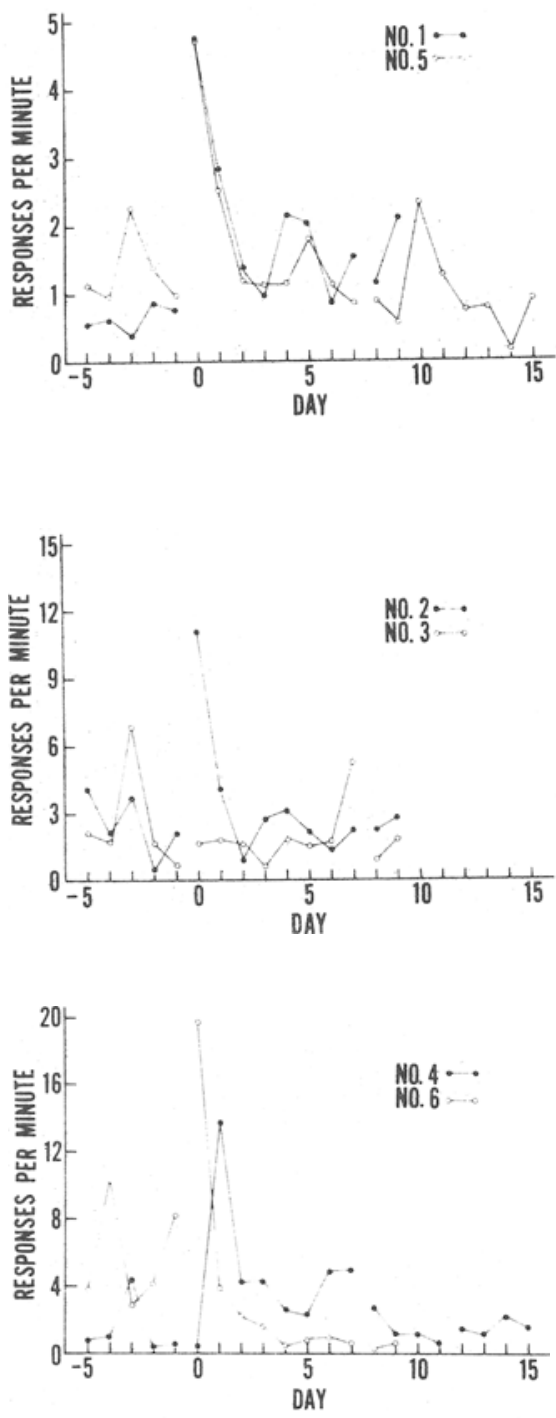

Fig. 1. Rate of response in $S 2$ by each rat over days.

previous day's level, increasing from 2.2 to 7.1 responses per minute. $\mathrm{S} 1$ mean response rate increased by only 1.2 responses per minute over those 2 days, so the $S 2$ increase did not reflect only day-to-day or general rate fluctuation. As Fig. 1 indicates, five of the six rats showed rate increases over these 2 days and the exception, Rat 4, whose rate was lowest of all Ss, showed the increase on the second day of this schedule. A t test comparing $S 2$ response rates on the last day of the original multiple schedule and the first day under the CRF:S condition yielded a value of $2.64, \mathrm{df}=5, \mathrm{p}<.05$.

Figure 1 shows a clear extinction effect for each rat. $A$ t test comparing mean response rates on the last day under the original multiple schedule (Day -1 ) and the last day under the CRF:S schedule (Day 7) yielded a value of only .27 , suggesting no detectable effect of the noise stimulus by the 8 th day of its availability in $\mathrm{S} 2$.

The return to the original multiple schedule, with the EXT component in S2, suggested no appreciable changes in the baseline rates for most rats. Rat 5 was continued the additional 6 days because of his downward trend over Days 8 and 9, a trend that proved inconsistent. Rat 4 demonstrated a baseline rate over Days 8 through 11 below his rate on the CRF:S schedule but, when returned to that schedule on Days 12 through 15, showed no tendency to increase responding with the noise again available.

\section{DISCUSSION}

The results indicate a substantial conditioned reinforcing effect of the noise on S2 response rates, an effect that extinguished quickly. The results were reasonably consistent across $\mathrm{Ss}$ and reached acceptable probability levels with only six Ss. The current procedure, then, permits a powerful test of conditioned reinforcing effects unconfounded by response strength and thus not requiring a cross-group comparison. While the conditioned reinforcer is not paired with primary reinforcement in the $S 2$ context, primary reinforcement is still available in $S 1$, so the general experimental situation maintains primary reinforcement and is not an extinction situation. Finally, the situation is analogous to nonexperimental situations in which conditioned reinforcement notions are used. Thus, the procedure seems a useful technique for the study of conditioned reinforcement effects.

In several studies reported after the completion of the present research, Thomas (1969) examined pigeons pecking a key for food on similar schedules. In one study, the first stimulus condition involved following every 120th peck with a brief stimulus and access to food (FR120), while in the second stimulus condition an extinction schedule was replaced by a schedule providing the brief stimulus, but no food, for every 10th response (FR10:S). Thomas's records indicate a dramatic increase in responding in the second stimulus condition, and he reported that such responding has been maintained in his laboratory for extended periods of time. Appropriate controls suggested that Thomas's results were a legitimate conditioned-reinforcement effect. The most obvious procedural differences between the current study and those of Thomas are the schedule of conditioned reinforcement during the second stimulus condition (CRF vs FR10) and the schedule of conditioned and primary reinforcement in the first stimulus condition (VI 20-sec vs FR120). Other studies reported by Thomas (1969) suggested that the more responses required to produce the conditioned reinforcer in the second condition, the weaker is its effect. Further, he varied the schedule of primary reinforcement in the first stimulus condition with no observable effect on responding for the conditioned reinforcer in the second condition. Consequently, the most likely explanation for the discrepant results of his research and the present study appears to involve the species difference.

Zimmerman (1969) has summarized a series of studies indicating that, in pigeons, responding can be maintained indefinitely with the only reinforcement an occasional brief stimulus which, though paired with food when automatically delivered, is never paired with food when response-produced. He has suggested the necessity of cross-species examination of his own results, and the same necessity exists in the present situation. The apparent lack of susceptibility of a conditioned reinforcer to a signaled extinction condition in Thomas's work, and to a consistent extinction contingency when response-produced in Zimmerman's work, demands determination of the cross-species generality of their findings. When viewed as a first step toward the extension of Thomas's results, the present data are less than encouraging. When viewed as exemplifying an additional technique for studying conditioned reinforcement, however, the procedure and data are promising.

\section{REFERENCES}

FERSTER, C. B., \& SKINNER, B. F. Schedules of reinforcement. New York: Appleton-Century-Crofts, 1957.

KELLEHER, R. T. Chaining and conditioned reinforcement. In W. K. Honig (Ed.), Operant behavior: Areas of research and application. New York: Appleton-Century-Crofts, 1966. Pp. 160-212.

KELLEHER, R. T., \& GOLLUB, L. R. A review of positive conditioned reinforcement. Journal of the Experimental Analysis of Behavior, 1962, 5, 543-597.

SKINNER, B. F. The behavior of organism \& New York: Appleton-Century-Crofts, 1938.

THOMAS, J. R. Maintenance of behavior by conditioned reinforcement in the signaled absence of primary reinforcement. In D. P. Hendry (Ed.), Conditioned reinforcement. Homewood, Ill: Dorsey Press, 1969. Pp. 77-90.

ZIMMERMAN, J. Meanwhile ... back at the key: Behavior sustained with conditioned reinforcement in an intermittent free-feeding situation. In D. P. Hendry (Ed.), Conditioned reinforcement. Homewood, Ill: Dorsey Press, 1969. Pp. 91-124.

ZIMMERMAN, J., \& HANFORD, P. V. Sustaining behavior with conditioned reinforcement as the only response-produced consequence. Psychological Reports, 1966, 19, 391-401.

\section{NOTE}

1. This technique was concurrently explored by J. R. Thomas (1969). The historical precedents of the present work did not include Thomas's research and seem still worthy of discussion. 